\title{
Entropy-based reliable design of water distribution networks
}

\author{
J. J. O. Sousa ${ }^{1}$, M. C. Cunha ${ }^{2}$ \& J. A. Sá Marques ${ }^{2}$ \\ ${ }^{1}$ Polytechnic Institute of Coimbra, Portugal \\ ${ }^{2}$ University of Coimbra, Portugal
}

\begin{abstract}
Water distribution networks are crucial for the wellbeing of communities, but their construction involves huge investment. The need to optimize investments has resulted in the development of methodologies to determine the minimum cost design of these infrastructures. However, as cost minimization tends to eliminate any redundancy in the networks, this kind of approach leads to less reliable solutions. From the reliability point of view, an ideal water distribution network should ensure reasonable service levels even when a failure occurs. But such a scenario, if possible, would certainly imply an intolerable level of investment. The following question therefore arises: by how much is it reasonable to increase investment to reduce the risk of failure? Trying to help decision makers answer this question, this work offers a tool to solve the reliable design of water distribution networks problem. It is based on an optimization model comprising two antagonistic objectives: minimizing the cost and maximizing reliability (here indirectly evaluated by the network entropy). The multiobjective problem is solved by the constraint method. The result is a set of cost minimization problems, each constrained by a different minimum level of entropy, which are solved by the Simulated Annealing method. This approach leads to a set of solutions known as Pareto solutions. Each of these solutions represents a different level of compromise between cost and reliability, and they can be very helpful for decision makers.
\end{abstract}

Keywords: water distribution networks, optimization, simulated annealing, reliability, entropy. 


\section{Introduction}

"Nothing in this world is 100\% reliable, and, as water engineers are painfully aware, urban water supply systems are no exception. ... In planning, engineers try to minimize the probability of failure, but $100 \%$ reliability is impossible. ... the heart of the design problem is to decide where along the reliability-cost frontier the system should be."

These statements, from Hobbs and Beim [2], may have been written almost two decades ago, but they especially illustrate a problem that remains very much one of today: the analysis of water supply systems' reliability. The reliability of these systems may be affected by several factors, namely: changes in water availability at source; changes in water quality at source; errors in consumption predictions used in the design and in the operation; failures in system components (treatment stations, reservoirs, pipes, pumps, valves); operational errors in defining necessary storage capacity to face peak consumption.

In general, reliability is defined as the probability that a system will perform well for a certain time when subject to specified conditions. In the sphere of water distribution network operations, reliability is related to the capacity to meet water consumption needs with pressure conditions that satisfy minimum requirements. The concept of fault is famously associated with reliability. In the present context, fault means the occurrence of a situation that impairs the network's ability to provide enough discharge, or sufficient pressure, to meet water demands, and from the evaluation point of view, water distribution networks reliability is affected by different kinds of fault: errors in water demand prediction, mechanical faults and faults following accidents.

The most usual way to assign reliability is by introducing some redundancy into the network. In the traditional design of water distribution networks, reliability is introduced by the application of some empirical rules, viz.: overestimation of pipe diameters, introduction of additional pipes to form loops, equipping pumping stations with emergency pumps, etc. In the context of optimal design, the reliability problem has been tackled in several ways in the last twenty years, and has given rise to a wide range of methodologies whose goal is to obtain optimal solutions (from the economics point of view) that are simultaneously reliable. The reliability of a network can be evaluated by analytical methods (based on probabilistic measures derived from graph theory) or by simulation methods (using simulators to estimate the network behaviour under fault scenarios and evaluating reliability from the results of the simulations performed). The diversity and complexity of situations they can deal with means that simulation methods are more likely to produce realistic analyses. Notwithstanding the efforts made in the last two decades, today there is still no universally accepted criterion to evaluate water distribution network reliability, and this situation constitutes a serious impediment to the progress of research in this sector. Most of the criteria proposed in the literature rely on the following: measures related to network topology; measures involving simultaneously the network topology and pipe capacities; deterministic and/or probabilistic 
measures related to the causes of network faults; measures based on the quantification of consumptions that cannot be totally supplied; resilience measures.

Concerning all these criteria, there are three major aspects that must be pointed out: some criteria are easy to implement but not rigorous enough to evaluate reliability; others are too demanding in terms of data needed for implementation; lastly, it is important to mention that, in the case of the most rigorous criteria, the evaluation of network reliability is by itself an NP-hard or $N P$-complete problem, in other words, a complex problem to tackle.

Nowadays one of the most promising measures to evaluate water distribution network reliability seems to be the maximization of the network entropy, and so this is the criterion adopted in this work.

\section{Reliable design of water distribution networks: entropy}

The use of the entropy concept to evaluate water distribution network reliability is based on two main principles: it is desirable for each node to be supplied by more than one pipe; pipes incident on each node should have similar capacities. The theoretical basis of this criterion is the principle of Shanon's entropy maximization, proposed several decades ago and used widely in the most varied areas of knowledge. Tanyimboh and Templeman [4] presented a way to evaluate entropy in water distribution networks. This approach relies on a multiple probability space model and the conditional entropy formula of Khinchin. Later, Walters [5] simplified the original expression and obtained a simpler one:

$$
\frac{E}{K}=-\sum_{i j \in M} \frac{Q_{i j}}{Q T_{0}} \cdot \ln \left(\frac{Q_{i j}}{Q T_{0}}\right)+\sum_{n=1}^{N} \frac{Q S_{n}}{Q T_{0}} \cdot \ln \left(\frac{Q S_{n}}{Q T_{0}}\right)
$$

where: $E$ : entropy;

$K$ :arbitrary positive constant (in the present context $K$ has no significant meaning such that it is usual to call network entropy by the relation $E / K$ );

$M$ :set of all positive flow links $i j$, including external inflows and outflows;

$Q_{i j}\left\{\begin{array}{l}i=0 \text { e } j \neq 0 \text { - external flow entering node } j \\ i \neq 0 \text { e } j=0 \text { - external flow leaving node } j \\ i \neq 0 \text { e } j \neq 0 \text { - flow in the pipe connecting nodes } i \text { and } j ;\end{array}\right.$

$Q T_{0}$ : total supply or demand of the network;

$N$ : set of junction nodes in the network;

$Q S_{n}$ : outflows at node $n$, including the node consumption.

This criterion to evaluate water distribution network reliability has two characteristics that make it very attractive, which are: it is not too demanding, and can be easily incorporated into optimization models. There are three different ways to incorporate this criterion into optimization models: first, by formulating an economical design model constrained by a minimum level of 
reliability, $E / K$; second, by formulating a reliability maximization model constrained by a budget, and finally by applying the Linear Programming method proposed by Alperovits and Shamir [1] to design the network subject to the flows that maximize its entropy. In most of the models to optimize the design of water distribution networks reliability is introduced as an additional set of constraints whose goal is to guide the design in order to obtain reliable solutions. The reliability constraints usually include some redundancy in the connections between reservoirs and consumption nodes to assure minimum service levels even in fault scenarios. This being so, and taking into account the enormous flexibility showed by modern heuristics in incorporating any kind of constraints, the methodology proposed by Cunha and Sousa [3], based on the Simulated Annealing method (see flowchart in Figure 1), was adapted to solve the reliable design of water distribution networks problem.

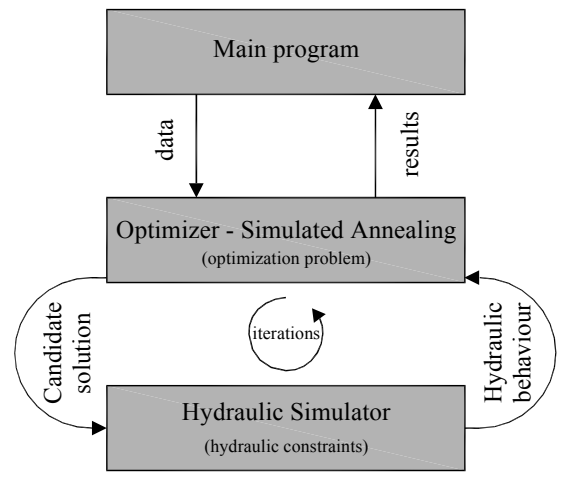

Figure 1: Flowchart explaining the methodology developed to solve the optimal design of water distribution networks.

The adaptation was restricted to the implementation of a subroutine that uses Eq. (1) to evaluate the entropy of each candidate solution. The reliable design of water distribution networks proceeds as follows: establish a minimum admissible level for the entropy, $(E / K)_{\min }$; throughout the search, after the hydraulic simulation of each candidate solution, the new subroutine is summoned to evaluate the entropy; if the entropy value surpasses the minimum required the solution is feasible; otherwise, the solution can be immediately rejected or accepted with a penalty as a consequence of the minimum entropy constraint violation. Meanwhile, the rest of the optimization process remains unchanged. The optimization model used in this work is the following:

$$
\text { Minimize } \sum_{j=1}^{N P} C_{p i p e, j}\left(D_{j}\right) \cdot L_{j}
$$

subject to:

$$
\sum_{j=1}^{N P} I_{i j} \cdot Q_{j}=Q c_{i} \quad i=1,2, \ldots, N
$$




$$
\begin{gathered}
\Delta H_{j}=\frac{10.674 L_{j}}{C_{H W j}^{1.852} \cdot D_{j}^{4.87}} \cdot Q_{j}^{1.852} \quad j=1,2, \ldots, N P \\
H_{i} \geq H_{i, \text { min }} \quad i=1,2, \ldots, N \\
D_{j}=\sum_{i=1}^{N D_{j}} Y D_{j, i} \cdot D C_{j, i} \quad \text { with } \sum_{i=1}^{N D_{j}} Y D_{j, i}=1 \quad j=1,2, \ldots, N C \\
E / K \geq(E / K)_{\min }
\end{gathered}
$$

where:

$N P$ : number of pipes in the network; $N$ : number of junction nodes in the network; $L_{j}$ : length of pipe $j(\mathrm{~m}) ; D_{j}$ : diameter of pipe $j(\mathrm{~m}) ; D C_{j, 1}, D C_{j, 2}, \ldots$, $D C_{j, N D}$ : set of commercial diameters for pipe $j(\mathrm{~m}) ; N D_{j}$ : number of commercial diameters in the set of pipes $j ; Y D_{j, i}$ : binary variables that identify the optimal commercial diameter for pipes $j ; C_{\text {pipe, } j}\left(D_{j}\right)$ : unit cost of pipe $j$ as a function of the diameter $(€ / \mathrm{m}) ; I$ : network incidence matrix $(N \mathrm{x} N P) ; Q_{j}$ : discharge in pipes $j$ $\left(\mathrm{m}^{3} / \mathrm{s}\right) ; \Delta H_{j}$ : headloss in pipes $j(\mathrm{~m}) ; C_{H W j}$ : Hazen-Williams coefficient of pipes $j$; $Q c_{i}$ consumption at junction node $i\left(\mathrm{~m}^{3} / \mathrm{s}\right) ; H_{i}$ : piezometric head at node $i(\mathrm{~m})$; $H_{i, \text { mim }}$ : minimum piezometric head for junction node $i(\mathrm{~m}) ; E / K$ : network entropy; $(E / K)_{\text {min }}$ : minimum level of network entropy.

This optimization model is nonlinear and the decision variables are discrete (binary variables that identify the optimal commercial diameters for the pipes).

\section{Application}

This section sets out to illustrate the procedure proposed to undertake the reliable design of water distribution networks. The optimization problem that must be solved has two objectives: cost minimization and reliability maximization. The methodology used here to measure the reliability is based on the entropy concept, $E / K$, evaluated by Eq. (1). Therefore, the reliability maximization is represented by the entropy maximization. This two-objective optimization problem is solved by the constraint method, by which one of the objectives is replaced by a constraint imposing a minimum level for that objective. In the present case the original two-objective problem gave way to a set of cost minimization problems, each one constrained by a different minimum level of entropy: $E / K \geq(E / K)_{\min }$. The application of this procedure is illustrated with the design of a new water distribution network (Figure 2) taken from $\mathrm{Xu}$ and Goulter [6].

This network is supplied by a single reservoir and has 33 pipes and 16 nodes. To achieve the design it was necessary to specify a set of commercial diameters whose unit costs were known. However, as in the methodology of $\mathrm{Xu}$ and Goulter [6] the diameters were continuous variables, the original version of this example only provided a cost function to evaluate the unit cost for each diameter value. In the absence of a set of commercial diameters, it was decided to use a ductile iron pipes set, with unit costs evaluated by the cost function from the original example (Table 1). 


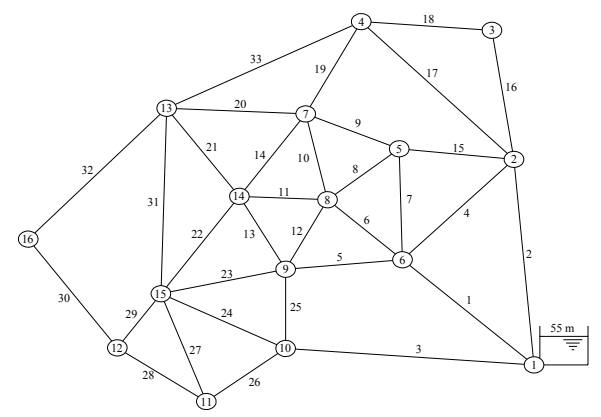

Figure 2: $\quad$ Scheme of the network.

Table 1: Available diameters and corresponding unit costs.

\begin{tabular}{c|c}
\hline $\begin{array}{c}\text { Diameter } \\
(\mathbf{m m})\end{array}$ & $\begin{array}{c}\text { Unit cost } \\
\mathbf{( \$ / \mathbf { m } )}\end{array}$ \\
\hline 100 & 12.052 \\
125 & 16.881 \\
150 & 22.231 \\
200 & 34.326 \\
250 & 48.079 \\
\hline
\end{tabular}

\begin{tabular}{c|c}
\hline $\begin{array}{c}\text { Diameter } \\
(\mathbf{m m})\end{array}$ & $\begin{array}{c}\text { Unit cost } \\
\mathbf{( \$ / \mathbf { m } )}\end{array}$ \\
\hline 300 & 63.317 \\
350 & 79.911 \\
400 & 97.763 \\
450 & 116.793 \\
500 & 136.933 \\
\hline
\end{tabular}

\begin{tabular}{c|c}
\hline $\begin{array}{c}\text { Diameter } \\
(\mathbf{m m})\end{array}$ & $\begin{array}{c}\text { Unit cost } \\
\mathbf{( \$ / \mathbf { m } )}\end{array}$ \\
\hline 600 & 180.332 \\
700 & 227.595 \\
800 & 278.439 \\
900 & 332.637 \\
1000 & 390.000 \\
\hline
\end{tabular}

After assembling all the necessary data, the reliable design process could be started. There was only one small dilemma: as the magnitude of the entropy values was not known a priori, what value should be specified for the minimum level to be imposed on the design? It is suggested that this difficulty may be overcome by using a preliminary design imposing $(E / K)_{\min }=0$, that is, relaxing the minimum entropy constraint. Depending on how the network entropy is evaluated, the result of this procedure is equivalent to a minimum cost design, and so a branched network solution should be obtained (any branched solution would present the same entropy value, which is precisely the minimum value it can take). The result of this design was, in effect, a branched network, costing $\$ 2.781 \times 10^{6}$ and with entropy equal to 2.708 (this solution is presented in Figure 3 and in Table 2). This minimum entropy value was then used as a reference to establish the minimum level of entropy to impose for each design, $(E / K)_{\min }$, and the design was repeated, but now with the entropy constraint active.

The goal of this work is to identify the solutions that optimize both objectives: economy (cost minimization) and reliability (entropy maximization). The solutions were obtained after executing several minimum cost designs, each one subject to a different minimum entropy level, starting from 2.708. The result of this procedure is a set of solutions (Pareto solutions) given in Figure 4, where a virtual line can be seen connecting them. This line separates the feasible region from the non-feasible region, that is: any feasible solution will be represented by a point over the virtual line (Pareto solution) or above that line; any point under the virtual line must represent a non-feasible solution (violates at least one of the problem's constraints). Table 2 presents some of the Pareto solutions obtained for this example, whose graphical representations can be seen in Figure 3. 
To facilitate the reading of Figure 3, the lines' thicknesses are proportional to the diameters of the pipes they represent.

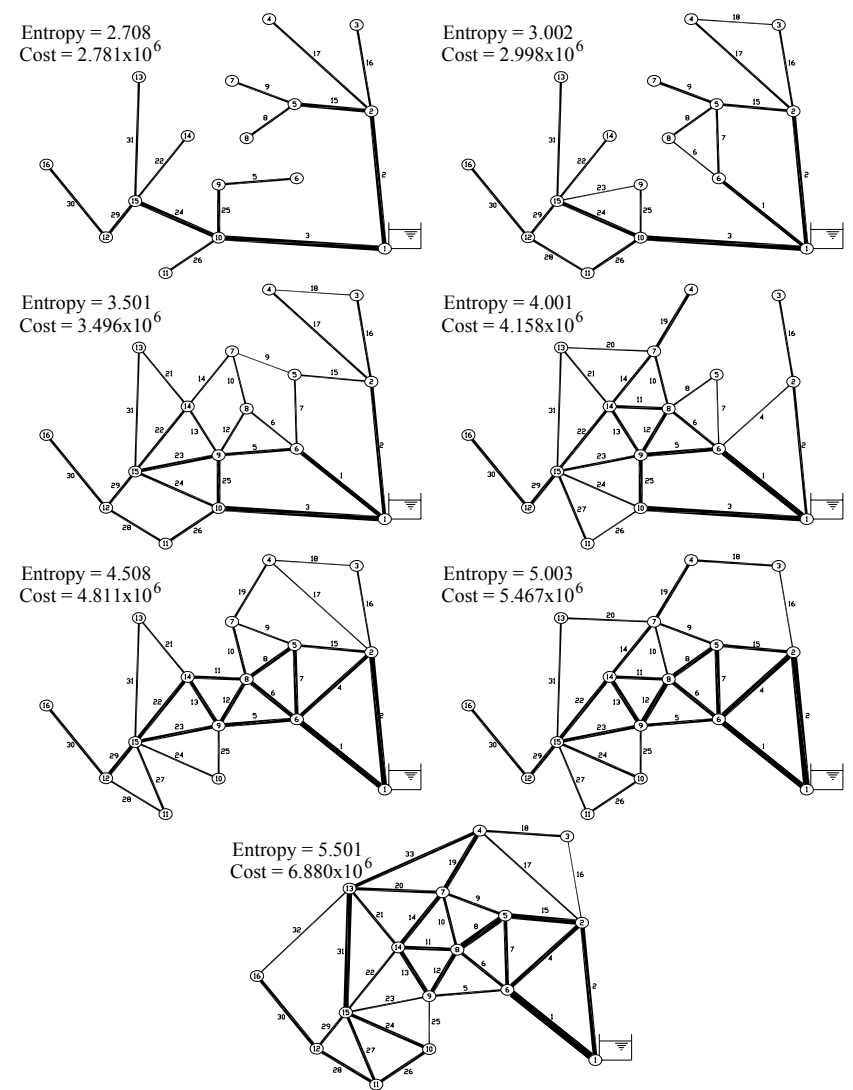

Figure 3: Design solutions for different levels of network entropy.

The analysis of these solutions leads to the following conclusions:

- the increase in the entropy was followed by an increase in the number of loops in the network. Keeping in mind that loops are a synonym of redundancy, and redundancy contributes to reliability, this result can be viewed as a demonstration of the relation between entropy and reliability;

- the increase in the entropy resulted in the increase in the diameters for the pipes located in the vicinity of the reservoir and in centre of the network. This phenomenon can also be viewed as an increase in reliability. As the diameters of the main pipes get bigger, the flexibility of the network will improve, and so the negative impacts resulting from an eventual failure of one of those pipes will be minimized;

- for the higher entropy levels the network is supplied only by two pipes leaving the reservoir. This result can be a direct consequence of the 
increase in diameters mentioned above, that is, the diameters of these pipes are so big that, most probably, if one of them fails the supply will be assured by the other.

Table 2: $\quad$ Design solutions for different levels of network entropy.

\begin{tabular}{c|c|c|c|c|c|c|c}
\hline Pipe & \multicolumn{7}{|c}{ Diameter (mm) } \\
\hline 1 & 0 & 350 & 450 & 600 & 600 & 600 & 800 \\
2 & 500 & 450 & 400 & 300 & 600 & 700 & 500 \\
3 & 600 & 600 & 600 & 600 & 0 & 0 & 0 \\
4 & 0 & 0 & 0 & 150 & 450 & 600 & 500 \\
5 & 250 & 0 & 350 & 450 & 450 & 350 & 250 \\
6 & 0 & 150 & 200 & 300 & 500 & 450 & 350 \\
7 & 0 & 250 & 200 & 150 & 450 & 500 & 400 \\
8 & 250 & 250 & 0 & 200 & 450 & 500 & 700 \\
9 & 250 & 300 & 100 & 0 & 200 & 250 & 300 \\
10 & 0 & 0 & 200 & 250 & 300 & 200 & 300 \\
11 & 0 & 0 & 0 & 350 & 350 & 350 & 400 \\
12 & 0 & 0 & 250 & 400 & 450 & 600 & 500 \\
13 & 0 & 0 & 250 & 350 & 450 & 500 & 500 \\
14 & 0 & 0 & 200 & 300 & 0 & 350 & 500 \\
15 & 450 & 300 & 200 & 0 & 300 & 350 & 600 \\
16 & 250 & 250 & 250 & 250 & 200 & 150 & 100 \\
17 & 250 & 250 & 250 & 0 & 150 & 0 & 200 \\
\hline
\end{tabular}

\begin{tabular}{c|c|c|c|c|c|c|c}
\hline Pipe & \multicolumn{7}{|c}{ Diameter (mm) } \\
\hline 18 & 0 & 125 & 100 & 0 & 125 & 250 & 250 \\
19 & 0 & 0 & 0 & 350 & 250 & 350 & 500 \\
20 & 0 & 0 & 0 & 150 & 0 & 200 & 350 \\
21 & 0 & 0 & 200 & 200 & 200 & 0 & 300 \\
22 & 250 & 250 & 300 & 350 & 450 & 450 & 250 \\
23 & 0 & 125 & 400 & 300 & 400 & 400 & 200 \\
24 & 500 & 450 & 300 & 200 & 200 & 300 & 400 \\
25 & 350 & 200 & 400 & 450 & 200 & 200 & 150 \\
26 & 250 & 300 & 300 & 150 & 0 & 250 & 350 \\
27 & 0 & 0 & 0 & 250 & 250 & 200 & 350 \\
28 & 0 & 250 & 250 & 0 & 200 & 0 & 350 \\
29 & 350 & 300 & 300 & 400 & 450 & 400 & 300 \\
30 & 300 & 300 & 300 & 350 & 300 & 300 & 400 \\
31 & 250 & 250 & 200 & 200 & 200 & 200 & 600 \\
32 & 0 & 0 & 0 & 0 & 0 & 0 & 150 \\
33 & 0 & 0 & 0 & 0 & 0 & 0 & 400 \\
\hline Entropy & 2.708 & 3.002 & 3.501 & 4.001 & 4.508 & 5.003 & 5.501 \\
\hline Cost (10 \$) & 2.781 & 2.998 & 3.496 & 4.158 & 4.811 & 5.467 & 6.880 \\
\hline
\end{tabular}

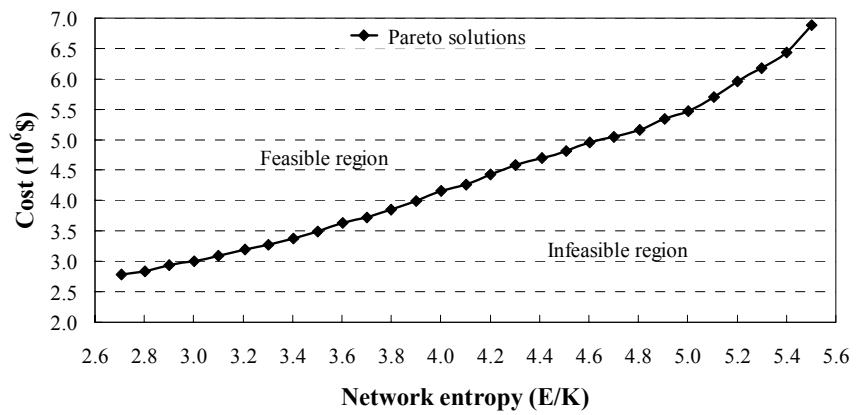

Figure 4: $\quad$ Pareto solutions (cost/entropy).

In the end, these solutions were compared with those arising from the economical design of the network, imposing different minimum pipe diameters, a procedure usually used as a means of conferring some level of reliability on the economical design. The minimum cost design was then found, imposing different minimum values for the pipes diameters, $D_{\min }$, namely: $D_{\min }=0$ (pure economical design), $100 \mathrm{~mm}, 125 \mathrm{~mm}, 150 \mathrm{~mm}, 200 \mathrm{~mm}$ and $250 \mathrm{~mm}$. After achieving the designs, the entropy values were evaluated and these solutions were compared with those obtained before (Figure 5), leading to the following conclusions:

- the increase in the minimum diameter value was followed by an increase in the entropy; 
- as the value of $D_{\min }$ increases the results deviate considerably from those obtained by the entropy procedure (the imposition of higher values for $D_{\min }$ results in overdesign of some pipes, mainly in the periphery of the network, and to the consequent cost increase);

- except for the solution obtained with $D_{\min }=0$, which is equivalent to $(E / K) \min =0$, the solutions obtained for different values of $D_{\min }$ present costs considerably higher than those obtained with similar entropy levels.

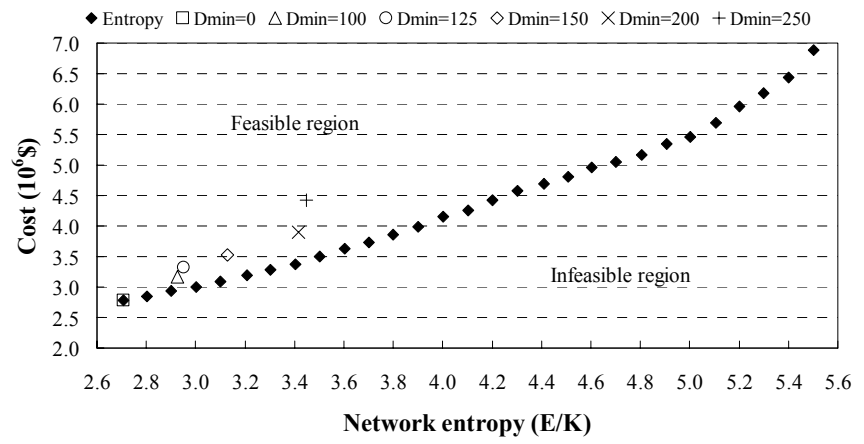

Figure 5: Minimum entropy constraints vs. minimum diameter constraints.

In the light of the above we can conclude that the imposition of minimum diameters can indeed be viewed as a contribution to increased network reliability, but it is not certain to be the most appropriate procedure to attain that goal.

\section{Conclusions}

This work tackles the reliable design of water distribution networks. The reliable design problem is transformed in an optimization problem with two objectives: minimization of cost and maximization of the reliability (here indirectly evaluated by the entropy, one of the most promising measures to evaluate water distribution network reliability). This problem is solved by the constraint method, by which one of the objectives is replaced by a constraint imposing a minimum level for that objective. In the present case the original two-objective problem gave way to a set of cost minimization problems, each constrained by a different minimum level of entropy. These problems were solved by the Simulated Annealing method.

The applicability of this methodology was illustrated with an example taken from the literature. The process begins by solving the cost minimization problem without any minimum entropy constraint, which resulted in a branched network, as expected. This solution presents the minimum value for the entropy, which is subsequently used as a reference. The problem is then solved again imposing different higher minimum levels for the entropy. This procedure yields a set of solutions, called Pareto solutions, arising from different levels of compromise 
between cost and reliability, which can be very helpful for decision makers. Analysis of the results shows that increased the entropy is followed by an increase in the network's redundancy, that is, its reliability. The Pareto solutions were compared with those arising from the economical design of the network imposing different minimum pipe diameters, a procedure normally used to confer some level of reliability on the economical design. This comparison showed that the imposition of minimum diameters does indeed contribute to an increase in reliability, but the solutions obtained with this procedure deviate considerably from the Pareto solutions, and therefore the use of this procedure is not advised.

\section{References}

[1] Alperovits, E., and Shamir, U. (1977). "Design of optimal water distribution systems." Water Resources Research, Vol. 13, No. 6, pp. 885-900.

[2] Hobbs, B.F., and Beim, G.K. (1988). "Analytical simulation of water system capacity reliability. 1. Modified frequency-duration analysis." Water Resources Research, Vol. 24, No. 9, pp. 1431-1444.

[3] Cunha, M.C., and Sousa, J.J.O. (1999). "Water distribution network design optimization: Simulated Annealing approach." Journal of water Resources Planning and Management, ASCE, Vol. 125, No. 4, pp. 215-221.

[4] Tanyimboh, T.T., and Templeman, A.B. (1993). "Calculating maximum entropy flows in networks." Journal of Operational Research Society, Vol. 44, No. 4, pp. 383-396.

[5] Walters, G.A. (1995). Discussion of "Maximum entropy flows in single source networks" de T.T. Tanyimboh, and A.B. Templeman. Engineering Optimization, Vol. 25, pp. 155-163.

[6] Xu, C., and Goulter, I.C. (1999). "Reliability-based optimal design of water distribution networks." Journal of Water Resources Planning and Management, ASCE, Vol. 125, No. 6, pp. 352-362. 